\title{
Facile fabrication of dendritic silver structures and their surface enhanced Raman spectroscopic properties
}

\author{
JISHENG YANG* and ZHENGDONG JIANG \\ School of Chemistry and Chemical Engineering, Yangzhou University, Yangzhou 225002, China \\ e-mail: jsyang@yzu.edu.cn
}

MS received 25 March 2014; revised 24 June 2014; accepted 25 June 2014

\begin{abstract}
A simple and efficient approach was developed to fabricate silver dendrites by $\mathrm{Cu}$ reducing $\mathrm{Ag}^{+}$ in $\mathrm{AgNO}_{3}$ solution. The growth speed, morphologies and structures of the silver dendrites strongly depend on $\mathrm{AgNO}_{3}$ concentration and reaction time. The silver dendrites were formed from nanosheets and the crystal structure is face-centered cubic. Rhodamine $6 \mathrm{G}$ was used as probe molecule to show that the silver dendrites have high sensitivity to surface enhanced Raman spectroscopy response.
\end{abstract}

Keywords. Dendritic silver; chemical synthesis; Raman spectroscopy.

\section{Introduction}

The nanoparticles of silver or certain other transition metals have received great attention due to their fascinating properties and intriguing applications that are complementary or superior to their bulk counterparts. ${ }^{1,2}$ It has been well-established that the physicochemical and optoelectronic properties of metal nanoparticles are size and shape dependent. Therefore, much attention has been focused on the size and shape controlled synthesis of metal nanoparticles. ${ }^{3,4}$ Accordingly, various shaped silver nanoparticles (Ag NPs) such as cubes,${ }^{5}$ rods, ${ }^{6}$ wires, ${ }^{7}$ disks, ${ }^{8}$ triangles ${ }^{9}$ and prisms $^{10}$ have already been synthesized using a variety of techniques. The dendritic or fractal Ag nanostructures have attracted the attention of scientists recently due to their attractive supramolecular structures, large surface area and excellent connectivity between the different parts of the structures. Significantly, it has been established that dendritic or fractal Ag nanostructures are an excellent material for surface enhanced Raman spectroscopy (SERS). ${ }^{11-14}$ Consequently, several efforts have been made to prepare dendritic Ag nanostructures using a variety of synthetic techniques. ${ }^{15-18}$ In these methods, however, it is not convenient to obtain high-purity $\mathrm{Ag}$ nanostructures due to the involvement of compounds which were used as reductants and structure-directors or stabilizers for preparing nanoparticles.

Since the first observation of SERS by Fleischmann et al. in $1974,{ }^{19}$ this technology has emerged as a routine and powerful tool for structural characterization

\footnotetext{
*For correspondence
}

of interfaces and molecularly thin-films. SERS is a technique that determines an enhanced Raman signal when a Raman-active molecule is in close proximity to an appropriate metallic surface, allowing detection of a few molecules and, in some cases, single molecule detection. Although the detailed mechanism of SERS enhancement has not been fully understood, SERS is generally explained by two different mechanisms: $:^{20}$ one is attributed to the local electromagnetic field at the metal surface or rough metal structures due to the surface plasmon polaritons, while the second is attributed to a chemical contribution due to an electronic resonance energy transfer between adsorbed molecules and the metal surface. Therefore, control over the composition, shape, size and local environment of surface structure is vital to achieve consistent SERS enhancement.

In this study, the dendritic silver was prepared by copper replacing silver ion in $\mathrm{AgNO}_{3}$ solution. The morphological evolution process of dendritic silver is shown in detail. Furthermore, application of the dendritic silver substrates in surface enhanced Raman scattering of Rhodamine 6G (Rh6G) is also demonstrated.

\section{Experimental}

\subsection{Chemicals}

Silver nitrate, Rh6G and ethanol were of analytical grade and used as received without further purification. The water used was deionized. Copper wire mesh was washed with acetone and ethanol in sequence, and dried in a vacuum at $60^{\circ} \mathrm{C}$. 


\subsection{Fabrication and characterization of dendritic silver structures}

A typical preparation of the dendritic silver structures on copper wire mesh was conducted as follows. First, a copper wire mesh (5 $\mathrm{mm}$ diameter) was polished with sandpaper, then washed with deionized water, and finally sonicated in deionized water for $2 \mathrm{~min}$ to remove surface debris and impurities. $20 \mathrm{mM} \mathrm{AgNO}_{3}$ solution was dripped on the surface of copper wire mesh at room temperature. The growth process of dendritic silver structures was observed by a DMLM/P light microscope (Leica, Germany) during the replacement reaction. The growth of silver dendrites was studied at different concentrations of $\mathrm{AgNO}_{3}$ under various reaction times. The resulting silver dendrites were washed with deionized water and absolute ethanol in sequence and dried by a stream of nitrogen gas. The silver dendrites were characterized by transmission electron microscopy (TEM, Tecnai-12, Philips Apparatus Co., USA) and X-ray diffraction (XRD, D8 Advance Super speed, Bruker-AXS Co., Germany).

\subsection{Raman measurements}

SERS measurement was performed on a Reinshaw Raman spectrometer (Renishaw plc, U.K.) by using a $\mathrm{He}-\mathrm{Cd}$ laser as the excitation light source at $785 \mathrm{~nm}$. Rh6G molecules were used to test the enhancement ability of the SERS active substrates. For SERS investigation, the SERS substrate was prepared by dropping the concentrated dispersion containing dendritic silver structures onto the $1 \times 1 \mathrm{~cm}^{2} \mathrm{Si}$ (111) wafer, which was allowed to dry naturally in air. Then, the substrates were soaked in $1.0 \times 10^{-8} \mathrm{M}, 1.0 \times 10^{-6} \mathrm{M}$ and $1.0 \times 10^{-4} \mathrm{M}$ Rh6G ethanol solution for $30 \mathrm{~min}$, respectively. After drying at room temperature, it was rinsed with ethanol thrice to remove free Rh6G molecules and dried in air. The whole SERS measurement was taken at room temperature.

\section{Results and Discussion}

\subsection{Formation of dendritic silver structure}

Low-magnification TEM picture (figure 1a) shows that the morphology of Ag trees presents a clear dendritic structure. High-magnification TEM picture (figure 1b) shows that the Ag dendrites were formed by a number of nanosheets and the length of each nanosheet is about $200 \mathrm{~nm}$. X-ray diffraction was performed to investigate the crystal structure. Figure $1 \mathrm{c}$ is the X-ray diffraction pattern of $\mathrm{Ag}$ dendrites. The five diffraction peaks can be indexed to diffraction from the (111), (200), (220), (311) and (222) of face-centered cubic (fcc) Ag (JCPDS Card File, 4-0783). The corresponding lattice constant calculated by XRD data is $0.40854 \mathrm{~nm}$, which is the same with that of $\mathrm{Ag}$ on the card.

\subsection{Effects of time of reaction on silver structures}

The growth process of dendritic silver structures in the replacement reaction can be clearly observed by a stereoscopic microscope. Figure 2 shows the morphologies in the continuous reactions of $\mathrm{Cu}$ and $\mathrm{AgNO}_{3}$ solution with a concentration of $20 \mathrm{mM}$ under the light microscope. Ag trees have grown out from the margin of copper wire with a height of $20 \mu \mathrm{m}$ for $2 \mathrm{~min}$ (figure 2a). Here branches of $\mathrm{Ag}$ trees are seldom, and only Ag trunks grow out quickly, which forms the dense branch morphology. At $5 \mathrm{~min}$, the roots of $\mathrm{Ag}$ tree still display dense branch morphology, and the tree tops begin to show a characteristic of fractal structure (figure 2b). After $10 \mathrm{~min}$, the Ag trees continue to grow
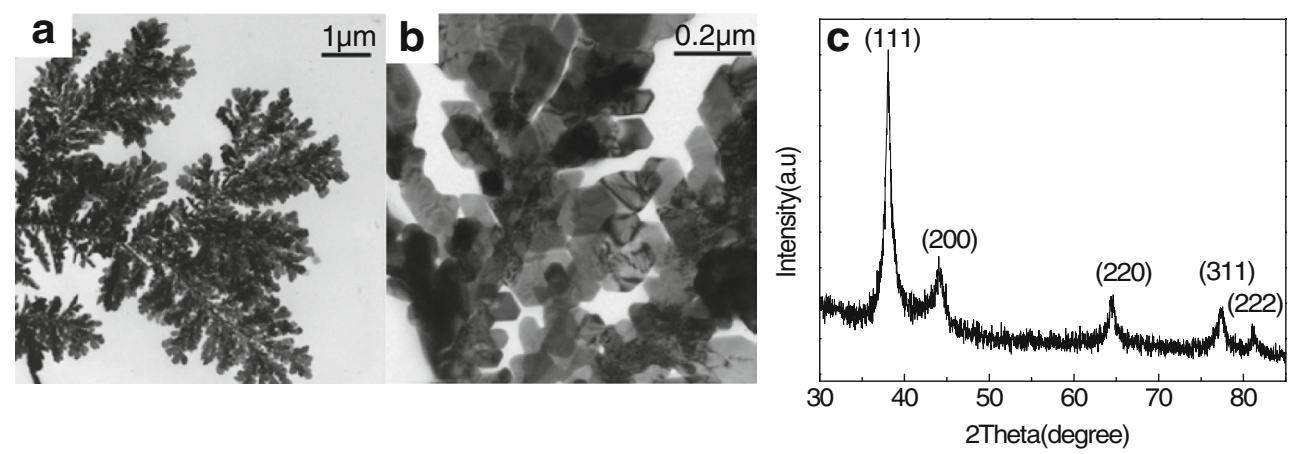

Figure 1. (a) Low magnification; (b) high magnification; (c) TEM images and XRD patterns of the silver trees synthesized after $10 \mathrm{~min}$ reaction of $\mathrm{Cu}$ and $100 \mathrm{mM} \mathrm{AgNO}_{3}$ solution. 

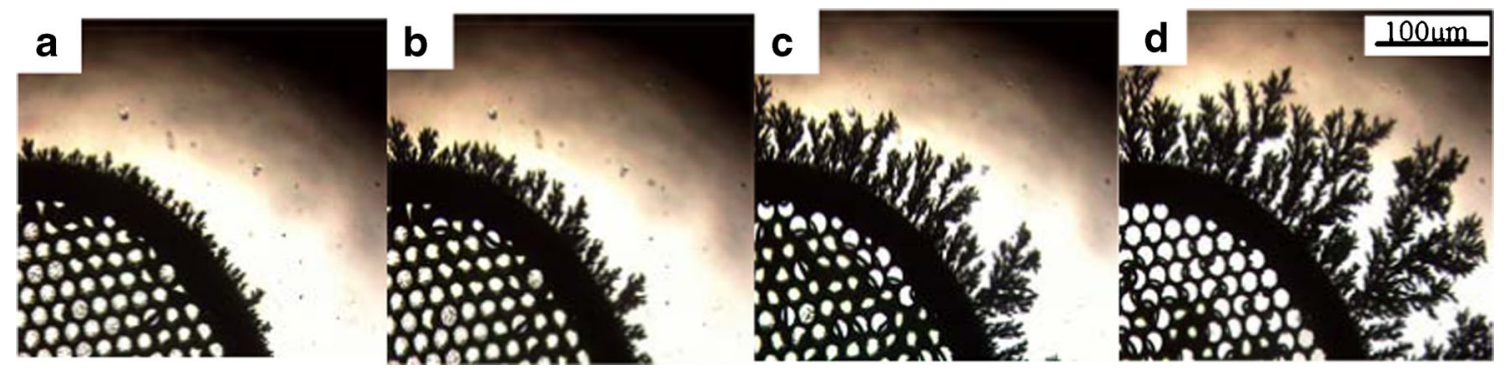

Figure 2. (a) Morphologies of silver trees grown in $20 \mathrm{mM} \mathrm{AgNO}$ solution for $2 \mathrm{~min}$; (b) $5 \mathrm{~min}$; (c) 10 $\min ;(\mathbf{d})$ and $20 \mathrm{~min}$.

to a height of $80 \mu \mathrm{m}$ and take on obvious fractal structure (figure $2 \mathrm{c}$ ). Figure $2 \mathrm{~d}$ is the $\mathrm{Ag}$ trees after 20 min with an obvious fractal structure and the height exceeds $120 \mu \mathrm{m}$. Although the detailed mechanism of the silver tree growth has not been fully understood, as literature has reported, ${ }^{21}$ we also speculate that the growth of silver tree involves the continuous deposition, spontaneous crystallization of amorphous phase and self-assembling of the crystallized nanocrystals. During the replacement reaction, the amorphous phase leads to continuous and rapid deposition on the surface of growing grains, and a number of silver nanograins with random orientation are crystallized spontaneously from the amorphous phase. The randomly oriented silver nanograins then realign and grow along a preferential direction and finally form a high-quality single crystal dendrite.

\subsection{Effects of $\mathrm{AgNO}_{3}$ concentration on the growth speed}

A series of $\mathrm{AgNO}_{3}$ solutions with concentration of 2, 10 and $100 \mathrm{mM}$ were used to explore the influence of $\mathrm{AgNO}_{3}$ concentration on the growth speed of dendritic silver structures. Figure 3 shows the change of $\mathrm{Ag}$ tree growth speed with different $\mathrm{AgNO}_{3}$ solution concentrations. From the figure, it can be seen clearly that the growth speed of Ag fractal structure mainly depends on the concentration of $\mathrm{AgNO}_{3}$ solution. The higher the concentration is, the faster it grows. From the overall trend, the $\mathrm{Ag}$ tree growth speed becomes faster and faster with the increase of concentration of $\mathrm{AgNO}_{3}$ solution, because $\mathrm{Ag}$ tree can capture $\mathrm{Ag}^{+}$more rapidly in relatively shorter mean free path during the growth process and reduce it into Ag.

\subsection{Effects of dendritic silver on the SERS of Rh6G}

The SERS sensitivity of the as-prepared Ag dendrites was investigated using $\mathrm{Rh} 6 \mathrm{G}$ as a model molecule. Figure 4 shows Raman spectrum of $1 \times 10^{-4} \mathrm{M}$ Rh6G ethanol solution adsorbed on a $\mathrm{Si}$ wafer (figure 4a) and the SERS spectra for different concentration of Rh6G ethanol solution adsorbed on $\mathrm{Ag}$ dendrites (figure 4b). Nearly no signals are observed in the spectrum of Rh6G adsorbed on Si wafer, while a number of intense peaks are seen in the spectra of figure $4 \mathrm{~b}$, which located at 1650, 1577, 1511, 1361, 1310, 1189, 1127,771 and $613 \mathrm{~cm}^{-1}$. Obviously, the SERS intensity is increased with increasing the Rh6G concentration adsorbed on Ag dendrites, and this dendritic Ag active substrate allows Rh6G to give Raman scattering also for diluted solutions $\left(10^{-8} \mathrm{M}\right)$. The bands between 1310 and $1650 \mathrm{~cm}^{-1}$ are due to the aromatic stretch vibrations of the chromophore, the peaks at 1511 and 1577 $\mathrm{cm}^{-1}$ which appear in ensemble spectra only as a shoulder arises from the $\mathrm{C}-\mathrm{O}-\mathrm{C}$ stretch vibration, the bands at 771 and $1127 \mathrm{~cm}^{-1}$ are the $\mathrm{C}-\mathrm{H}$ out of plane and in plane vibration, the line at $613 \mathrm{~cm}^{-1}$ is the C-C-C ring in plane vibration. It has been proposed that metal

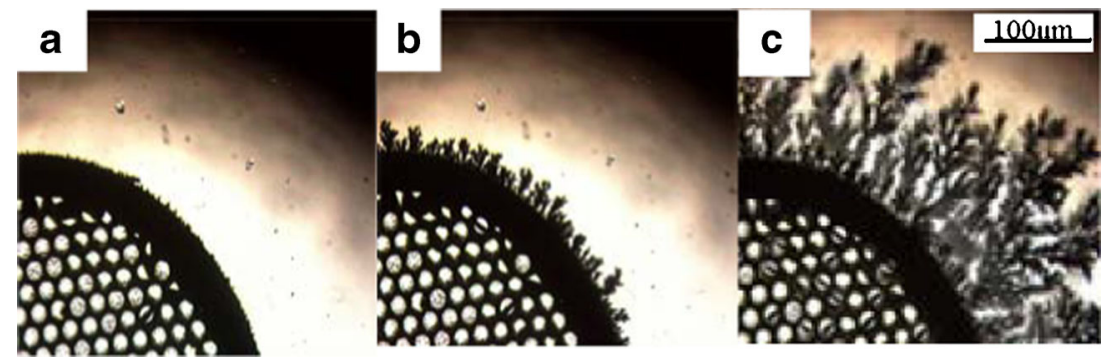

Figure 3. Morphologies of silver trees grown for $10 \mathrm{~min}$ in different concentration of $\mathrm{AgNO}_{3}$ solution: (a) $2 \mathrm{mM}$; (b) $10 \mathrm{mM}$; (c) $100 \mathrm{mM}$. 

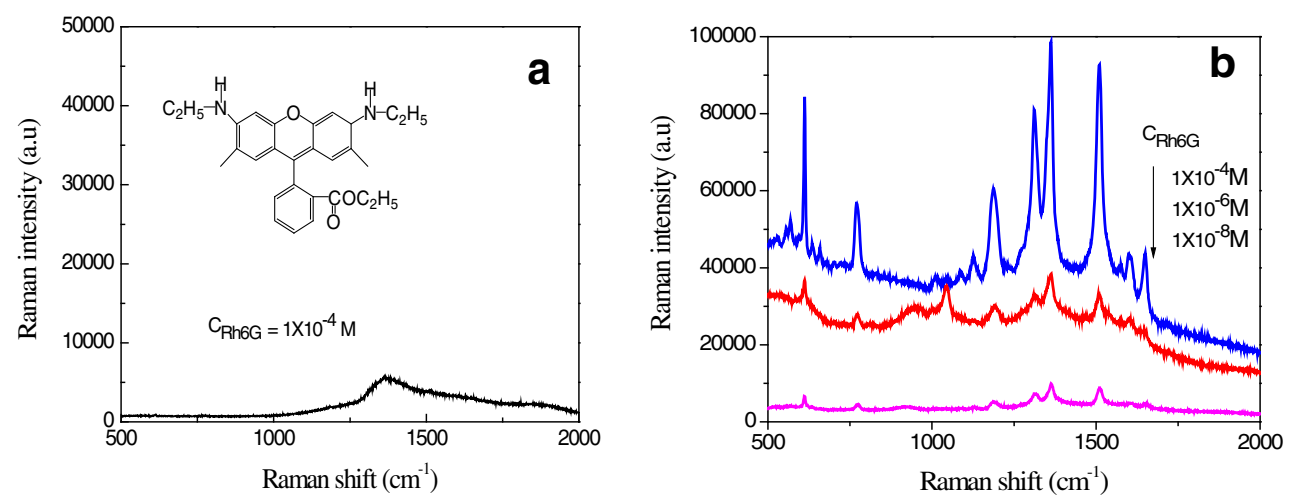

Figure 4. (a) Raman spectrum for Rhodamine 6G on a Si wafer; (b) SERS spectra for different concentration of Rhodamine $6 \mathrm{G}$ on the dendritic silver substrate.

nanostructures with sharp corners or edges exhibited excellent SERS properties. ${ }^{22}$ In the present case, the observed large enhancement in Raman scattering of the Rh6G molecules on the Ag dendrites consisting of nanosheets could be also ascribed to the giant local electromagnetic field due to the formation of many hot spots at sharp corners or edges of the sheets. Therefore, the obtained Ag dendrites could be used as an effective SERS substrate that may find many potential applications.

\section{Conclusions}

To summarize, a simple and efficient approach was developed to fabricate silver dendrites by the replacement reaction. The growth speed, morphologies and structures of the silver dendrites strongly depend on $\mathrm{AgNO}_{3}$ concentration and reaction time. The TEM and XRD results revealed that the silver dendrites were formed by nanosheets and the crystal structure of $\mathrm{Ag}$ dendrites is face-centered cubic. Rh6G was used as probe molecules for SERS experiments to show that this new silver active substrate has high sensitivity to SERS response.

\section{Acknowledgements}

We gratefully acknowledge the financial support from a project funded by the Priority Academic Program Development of Jiangsu Higher Education Institutions and devices support from testing center of Yangzhou University.

\section{References}

1. Schulz J, Roucoux A and Patin H 2002 Chem. Rev. 102 3757

2. Gopidas K R, Whitesell J K and Fox M A 2003 Nano Lett. 31757

3. Sun Y and Xia Y 2002 Science 2982176

4. Teng X and Yang H 2005 Nano Lett. 5885

5. Yu D and Yam V W W 2004 J. Am. Chem. Soc. 12613200

6. Chen H, Gao Y, Zhang H, Liu L, Yu H, Tian H, Xie S and Li J 2004 J. Phys. Chem. B 10812038

7. Sun Y, Mayers B, Herricks T and Xia Y 2003 Nano Lett. 3955

8. Maillard M, Huang P and Brus L 2003 Nano Lett. 31611

9. Chen S and Carroll D L 2002 Nano Lett. 21003

10. Jana N R, Gearheart L and Murphy C J 2001 J. Phys. Chem. B $\mathbf{1 0 5} 4065$

11. Hao E, Schatz G and Hupp J 2004 J. Fluoresc. 14331

12. Cobley C M, Skrabalak S E, Campbell D J and Xia Y 2009 Plasmonics 4171

13. Brejna P R, Sahaym U, Norton M G and Griffiths P R 2011 J. Phys. Chem. C 1151444

14. Orendorff C J, Gearheart L, Jana N R and Murphy C J 2006 Phys. Chem. Chem. Phy. 8165

15. Zhou Y, Yu S H, Wang C Y, Li X G, Zhu Y R and Chen Z Y 1999 Adv. Mate. 11850

16. Zhu J, Liu S, Palchik O, Koltypin Y and Gedanken A 2000 Langmuir 166396

17. Lee G J, Shin S I and Oh S G 2004 Chem. Lett. 33118

18. Jiang G H, Wang L, Chen T, Yu H J and Wang J J 2005 J. Mate. Sci. 401681

19. Fleischmann M, Hendra P J and McQuillan A J 1974 Chem. Phys. Lett. 26163

20. Eustis S and El-Sayed M A 2006 J. Appl. Phys. 100 044324

21. Fang J X, Ma X N, Cai H H, Song X P, Ding B J and Guo Y 2006 Appl. Phys. Lett. 89173104

22. Xia Y, Xiong Y, Lim B and Skrabalak S E 2009 Angew Chem. Int. Ed. $\mathbf{4 8} 60$ 